courses were admistred at normal times regularly, $60(30.9 \%)$ were slightly irregular and $32(16.5 \%)$ were irregular. There was no significant difference between the mean DAS-28 responses in the time of the courses and the rituximab infusion compliances [in regular group mean DAS-28 was $3.31(1,19)$, in slightly irregular group mean DAS-28 was 3.26 (1.35) and irregular group mean DAS-28 was 3,57 (1.34), $p>0.05$ ]. Before rituximab courses, $33.7 \%$ of patients had remission, $19.1 \%$ had low disease activity, $36.3 \%$ had moderate disease activity and $10.9 \%$ had high disease activity.

Conclusions: Rituximab administration was approximately 2 months delayed in RA patients who were living in center-city boundaries. The pre-treatment mean disease activity scores were similar, even if there was a delay in rituximab administration. In our center, about $85 \%$ of patients were taking RTX courses regular and/or slightly irregular and about half of the patients before treatment were in remission and/or low disease activity.

Disclosure of Interest: None declared

DOI: 10.1136/annrheumdis-2017-eular.4430

\section{AB0415 TENDENCY TO CHOOSE FIRST BIOLOGIC AGENT THERAPY OF RHEUMATOID ARTHRITIS IN THE ELDERLY: RESULTS FROM JAPANESE MULTICENTER REGISTRY}

T. Watanabe, T. Kojima, N. Takahashi, S. Asai, N. Asai, T. Matsumoto, Y. Sobue, N. Ishiguro. Orthopaedic Surgery and Rheumatology, Nagoya University Graduate School of Medicine Department of Orthopaedic Surgery, Nagoya, Japan

Background: Of the treatment of rheumatoid arthritis (RA), biologic agent therapies are chosen, if disease activity remains moderate or high despite csDMARDs therapy. In the elderly, with comorbidity and their less spare ability, safety is often concerned in the choice of biologic agent.

Objectives: We investigated the tendency to choose biologic agent and drug continuation rates in elderly in last decade.

Methods: Records of relevant patients with RA were collected from the Tsurumai Biologic Communication Registry, wherein the department of Nagoya University and 20 affiliated hospitals in Japan are enrolled. A total of 873 biologics-naïve and age 65 and older patients were recruited from January 2004 to December 2014. We studied the choice of the biologic agent year by, and baseline disease activity and concomitant methotrexate (MTX) among TNF inhibiters, tocilizumab (TCZ), and abatacept (ABT) groups. Drug continuation rates were compared among TNF inhibiters, TCZ, and ABT groups.

Results: From 2005 to 2010 , etanercept (ETN) was used the most (2007; $73.5 \%$, $2009 ; 65.9 \%)$. After the advent of ABT, ABT was used the most $(2011 ; 44.3 \%$, $2014 ; 38.8 \%$ )

Baseline disease activity slightly decreased as a whole (DAS28-CRP; 4.88 to 4.44). Despite baseline disease activity of TNF inhibitors group decreased (DAS28-CRP; 4.88 to 4.37), that of TCZ group increased (DAS28-CRP; 4.94 to $6.24)$

In 2011-2014, baseline disease activity of TCZ group (5.85) was higher than that of TNF inhibiters group $(5.11)(\mathrm{p}<0.05)$. Concomitant MTX rate and dose were lower in ABT group $(40.2 \%, 7.6 \mathrm{mg})$ than that of TNF inhibiters group $(79.0 \%$, $8.9 \mathrm{mg}$ ). 2 years drug continuation rate due to all unfavorable causes; ABT group was $92.4 \%$, better than that of TNF inhibitors $87.1 \%$ and TCZ 69.2\% $(p<0.05)$.

2 years drug continuation rate due to adverse events; TCZ group was $80.8 \%$, lower than that of TNF inhibitors $94.6 \%$ and ABT $96.2 \%(p<0.05)$

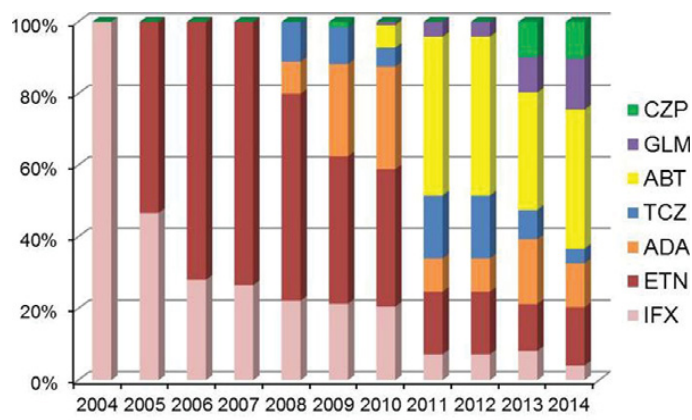

Figure 1. Changes of biologic agent from 2004 to 2014 (IFX:infliximab, ETN: etanercept, ADA: adalimumab,

T:

CZP:certolizumab pegol)

Conclusions: ETN was used most before the advent of ABT. After the advent of $A B T, A B T$ was used most and ETN decreased. This selection was made for speculation that $A B T$ is lower risk than other biological agents. Baseline disease activity slightly decreased showing that tight control management became also popular among elderly. In 2011-2014, concomitant MTX rate and dose were lower in ABT group, but 2 years drug continuation rate was the highest.

Disclosure of Interest: None declared

DOI: 10.1136/annrheumdis-2017-eular.1679

\section{AB0416 WHICH FACTORS PREDICT THE RESPONSIVENESS TO TOCILIZUMAB IN RHEUMATOID ARTHRITIS? THE DIFFERENCE BETWEEN THE USAGE AS THE FIRST BIOLOGIC AND AS THE SECOND BIOLOGIC}

Y. Kunishita, R. Yoshimi, H. Nagai, N. Hamada, Y. Soejima, Y. Sugiyama, N. Tsuchida, H. Nakano, D. Kishimoto, R. Kamiyama, Y. Asami, Y. Kirino, H. Nakajima. Department of Stem Cell and Immune Regulation, Yokohama City University Graduate School of Medicine, Yokohama, Japan

Background: Although recent development of a variety of biologics has dramatically improved treatment for rheumatoid arthritis (RA), it is still unclear which biologics is better for use in each patient. Some previous studies have shown the predictive factors for good response (GR) to tocilizumab (TCZ), including low $\mathrm{HAQ}$, high DAS28 ${ }^{1)}$, low levels of serum soluble IL-6 receptor ${ }^{2)}$, and low numbers of previous use of other biologics ${ }^{3)}$. However, the consensus is not immediately available.

Objectives: To compare continuation rates (CR) of TCZ by the responsiveness to the therapy and to identify predictive factors for GR to TCZ in RA.

Methods: Patient with RA who newly started receiving TCZ after April 2008 in our hospital, were included in the study. We collected patient records, medication histories, laboratory data, and clinical parameters longitudinally after starting TCZ. Statistical analyses were performed using the chi-square test, binomial logistic regression analysis, Kaplan-Meier method, and the log-rank test.

Results: Ninety-two patients were included in the study. The mean age and disease duration at baseline were $60.0 \pm 13.5$ years and $8.7 \pm 8.0$ years, respectively. The seroprevalence of the anti-cyclic citrullinated peptide antibody and the rheumatoid factor were $95.4 \%$ and $95.7 \%$, respectively. The rate of methotrexate and prednisolone at baseline were $45.7 \%$ and $64.1 \%$, respectively. TCZ was administered as the first biologic in 42 patients, and as the second biologic in 33. DAS28(ESR) and CDAI revealed high disease activity at baseline (5.2 \pm 1.5 and $25.4 \pm 14.1$, respectively). The mean CR of all patients was $42.1 \pm 4.0$ months. The CR was significantly higher in patients who achieved GR in EULAR response criteria at 6 months after starting TCZ than those who did not achieve GR (54.0 \pm 6.0 months vs $29.0 \pm 5.3$ months, $p=0.004$ ). Multivariate statistical analysis revealed two predictive factors for achieving GR at 6 months after starting TCZ, the low number of previous use of other biologics and the low CDAI at baseline $(p=0.018$, odds ratio $(\mathrm{OR})=0.386$, and $p=0.011$, $\mathrm{OR}=0.944$, respectively). We divided the patients into two groups, patients using TCZ as the first biologic and patients using it as the second biologic. Univariate statistical analyses revealed low usage rate and dose of prednisolone (PSL) and low serum creatinine level at baseline as the predictive factors for achieving GR in patients using TCZ as the first biologic, and low DAS28(ESR), CDAI and HAQ-DI in the patients using TCZ as the second biologic. By multivariate statistical analysis, we identified the low CDAl as a predictive factor in the patients using TCZ as the second biologic.

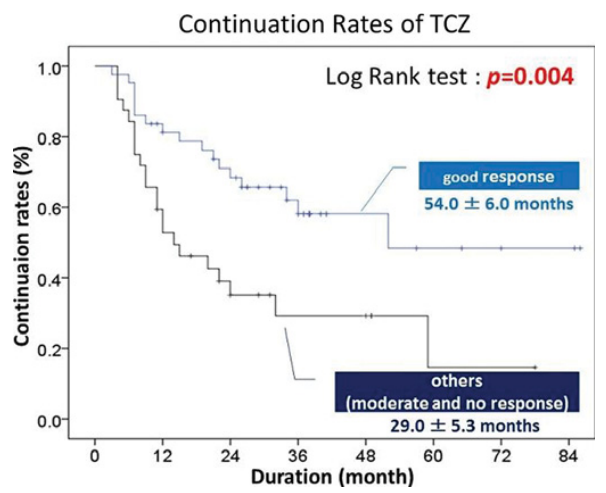

Conclusions: RA patients who achieved GR at 6 months after starting TCZ showed higher CR than the others. This study also suggests that low number of biologics usage and low CDAl at baseline are the predictive factors for GR. The history of biologics usage may be important to identify the predictive factors for GR to TCZ.

References:

[1] Ann Rheum Dis 2011;70:1216-22.

[2] Ann Rheum Dis 2014;73:945-7.

[3] Pharmacological Research 2016;111:264-71.

Disclosure of Interest: None declared

DOI: 10.1136/annrheumdis-2017-eular.4059 